\title{
ASPERSIÓN FOLIAR DE ÁCIDO SALICÍLICO INCREMENTA LA CONCENTRACIÓN DE FENOLES EN EL GRANO DE MAÍZ
}

\author{
LEAF SPRAYING OF SALICYLIC ACID INCREASES THE \\ CONCENTRATION OF PHENOLS IN MAIZE GRAIN
}

\section{César Tucuch-Haas ${ }^{1}$, Gabriel Alcántar-González ${ }^{1 *}$, Yolanda Salinas-Moreno², Libia I. Trejo-Téllez ${ }^{1}$, Víctor H. Volke-Haller ${ }^{1}$ y Alfonso Larqué-Saavedra ${ }^{3}$}

\begin{abstract}
'Postgrado en Edafología, Colegio de Postgraduados. km 36.5 Carr. Federal México-Texcoco. 56230, Montecillo, Texcoco, Estado de México, México. ${ }^{2}$ Campo Experimental Altos de Jalisco, Instituto Nacional de Investigaciones Forestales, Agrícolas y Pecuarias. km 8 Carr. Tepatitlán-Lagos de Moreno. 47600, Tepatitlán de Morelos, Jalisco, México. ${ }^{3}$ Recursos Naturales, Centro de Investigación Científica de Yucatán. Chuburná de Hidalgo Mérida. 97200, Yucatán, México.
\end{abstract}

*Autor para correspondencia (alcantar@colpos.mx)

\section{RESUMEN}

El ácido salicílico (AS) es una molécula multifuncional en las plantas. Se presentan los resultados del contenido total de fenoles solubles (FST) en el grano de maíz (Zea mays L.), cosechado de plantas que fueron asperjadas durante el estado de plántula con $0.101 \mu \mathrm{M}$ de ácido salicílico (AS). La cuantificación de los FST se realizó por el método de Folin-Ciocalteu. El tratamiento de $1 \mu \mathrm{M}$ de AS incrementó significativamente la concentración de FST en el grano en $17 \%$ comparado con el testigo. Los resultados se discuten en relación con la importancia de estos metabolitos secundarios como antioxidantes y compuestos de defensa contra los patógenos.

Palabras clave: Zea mays, ácido salicílico, fenoles, grano.

\section{SUMMARY}

Salicylic acid (SA) is a multifunctional molecule in plants. This paper shows results on the total content of soluble phenols (TSF) in maize (Zea mays L.) grain harvested from plants sprayed during the seedling stage with either 0.1 or $1 \mu \mathrm{M}$ SA. Quantification of TSF was performed by the Folin-Ciocalteu technique. Treatment of $1 \mu \mathrm{M}$ of SA significantly increased TSF concentration in the grain by $17 \%$ compared to the control. Results show the importance of these secondary metabolites as antioxidants and defense compounds against pathogens.

Index words: Zea mays, salicylic acid, phenols, grain.

\section{INTRODUCCIÓN}

Diversos trabajos señalan el potencial del ácido salicílico (AS) como regulador de procesos fisiológicos y bioquímicos en las plantas (Hayat et al., 2010), que favorecen el desarrollo (Tucuch et al., 2015; Tucuch-Haas et al., 2016), la bioproductividad (Martín-Mex et al., 2013) y la tolerancia a daños causados por factores bióticos (An y Mou, 2011) y abióticos (Khan et al., 2015). El AS ha sido asociado con la producción de compuestos fenólicos (Rodrigues-Brandao et al., 2014) como el ácido rosmarínico (Hao et al., 2014), ácido salvianólico B, ácido cafeico (Dong et al., 2010) y flavonoides (Pacheco et al., 2013); además de otros tipos de metabolitos secundarios como a y $\beta$ pineno, $\rho$-cimeno, a-tujeno, a-cadineno, cadinol, t-muurolol, 1, 8-cineol y limoneno (Al-oubaidi y Ameen, 2014).

Los fenoles representan un grupo amplio de compuestos característicos de las plantas, que entre otras funciones, juegan un papel importante en la pigmentación, crecimiento, reproducción y adaptación a condiciones de estrés biótico y abiótico (Farah y Donangelo, 2006; Lattanzio et al., 2006). Fisiológicamente los fenoles están involucrados en la absorción de nutrimentos, síntesis de proteínas, actividad enzimática, fotosíntesis, componentes estructurales y alelopatía (Robbins, 2003).

En el cultivo de maíz (Zea mays L.), los compuestos fenólicos participan en la coloración (Salinas et al., 2013), dureza (Cabrera-Soto et al., 2009) y tolerancia a patógenos en el grano (Bakan et al., 2003; García et al., 2003). Por otro lado, el consumo de granos ricos en fenoles trae consigo beneficios en la salud humana (Liu, 2007), debido a sus propiedades antioxidantes y anticancerígenas (Quiñones et al., 2012).

Dada la importancia de los compuestos fenólicos en las plantas y en la salud humana, se llevó a cabo la presente investigación en el cultivo de maíz, con el propósito de determinar el efecto del AS asperjado al dosel de plántulas de maíz sobre la concentración de fenoles en el grano.

\section{MATERIALES Y MÉTODOS}

Se establecieron dos experimentos independientes con maíz de la raza Xmejen-nal (Nal-tel × Tuxpeño) en suelo, bajo condiciones de campo, en los terrenos del Centro de 
Investigación Científica de Yucatán, ubicado en la ciudad de Mérida, Yucatán. El primero se estableció el 30 de agosto de 2013 y el segundo el 19 de enero de 2014 con el propósito de verificar la reproducibilidad del efecto. Las siembras se realizaron de forma directa en el suelo, en camas de $1 \mathrm{~m}$ de ancho $\times 10 \mathrm{~m}$ de largo, con distanciamientos entre plantas de $0.30 \mathrm{~m}$ y $0.50 \mathrm{~m}$ entre hileras, con una población total de 60 plantas, mismas que se distribuyeron de forma equitativa entre los tratamientos y el testigo, en ambos experimentos, bajo un diseño experimental completamente al azar con 20 repeticiones, en la que se tomó una planta como unidad experimental. Cabe señalar que para la cuantificación del contenido de fenoles, en cada experimento, se concentraron las muestras de las 20 repeticiones de cada tratamiento, de donde se tomaron cuatro muestras de $100 \mathrm{~g}$ por tratamiento, mismas que se analizaron estadísticamente como se señala más adelante.

Plantas de $7 \mathrm{~d}$ después de la emergencia, fueron asperjadas en la parte aérea con ácido salicílico (AS) a concentraciones de 0.1 y $1 \mu \mathrm{M}$ (Gutiérrez-Coronado et al., 1998) y como testigo se asperjó agua destilada. Se realizaron siete aplicaciones matutinas, hasta punto de goteo, en intervalos de $24 \mathrm{~h}$. Después de la última aspersión de AS, las plantas se dejaron crecer libremente, se realizaron deshierbes ocasionalmente, se llevaron a cabo riegos para mantener el suelo húmedo a capacidad de campo y una aplicación de fertilizantes, al suelo, de triple 17 (17N, 17P, $17 \mathrm{~K}), 20 \mathrm{~g}$ por planta a los $20 \mathrm{~d}$ después de la siembra (dds). A los 140 dds (momento de la cosecha) se colectaron muestras de granos, a los que se les midió la concentración de fenoles totales. Para la determinación, se tomaron cuatro muestras de 100 granos sanos de cada tratamiento, se molieron en un molino tipo ciclónico (Ika® Works, Inc. Wilmington, North Carolina, USA) con malla de $0.5 \mathrm{~mm}$ y se deshidrataron en una estufa (Binder, FED720, Tuttligen Alemania) a $50{ }^{\circ} \mathrm{C}$ por $12 \mathrm{~h}$. Se pesó $1 \mathrm{~g}$ de cada muestra y se agregaron $20 \mathrm{~mL}$ de metanol al $80 \%$ como disolvente. Las muestras se sometieron por 15 min a un baño de ultrasonidos y posteriormente se agitaron por 105 min en un agitador horizontal para realizar la extracción.

Las muestras se centrifugaron por 10 min a $2325 \times \mathrm{g}$ (centrifuga Hettich Zentrifugen, modelo Universal 32, Tuttligen, Alemania) y el sobrenadante se filtró con papel Whatman No. 4 y se midió su volumen. La cuantificación de los fenoles solubles totales (FST) se realizó por el método colorimétrico de Folin-Ciocalteu, de acuerdo con lo descrito por Makkar (2003). La detección de los compuestos fenólicos se realizó a $760 \mathrm{~nm}$ de absorbancia (espectrofotómetro Perkin Elmer ${ }^{\circledR}$ Lambda UV/VIS, Massachusetts, USA) y los resultados se expresaron en función del ácido gálico, para lo cual se elaboró una curva patrón con dicho estándar.
Con los valores de ambos experimentos se realizaron análisis de varianza, de manera individual por cada experimento, mediante el modelo lineal correspondiente a un diseño experimental completamente al azar; para la cuantificación y análisis estadístico del contenido de fenoles en el grano, para cada experimento, se concentraron las 20 repeticiones por tratamiento en una sola muestra, de donde se tomaron cuatro muestras de $100 \mathrm{~g}$ por tratamiento, de las que resultaron cuatro repeticiones para el análisis. Se realizó la comparación de medias mediante la prueba de Tukey $(P \leq 0.05)$ con el paquete estadístico SAS.

\section{RESULTADOS Y DISCUSIÓN}

En la Figura 1 se presentan los resultados de la concentración de FST de ambos experimentos. Se observa que la aspersión foliar de AS a una concentración de $1 \mu \mathrm{M}$ en la fase de plántula, incrementó significativamente la concentración de FST en grano, con una concentración media de $877.2 \mathrm{mg} \mathrm{g}^{-1}$ de materia seca, lo que representa en promedio una concentración superior $17 \%$ a la registrada en el testigo. La concentración $0.1 \mu \mathrm{M}$ de AS no fue estadísticamente diferente al tratamiento testigo en los dos experimentos; lo anterior a pesar de un incremento de $4 \%$ con respecto al testigo en el experimento uno.

Los resultados encontrados en la presente investigación indican que el AS aumentó la concentración de fenoles en el grano de maíz y son coincidentes con los encontrados en otras especies como aceituna (Simarouba glauca) (Awate y Gaikwad, 2014) y naranja agria (Citrus aurantium) (Sarrou et al., 2015), cuando se suministra el AS de manera foliar y en salvia roja (Salvia miltiorrhiza) cuando se aporta al medio en cultivo de tejidos (Dong et al., 2010). Dong et al. (2010) y Hao et al. (2014) sugieren que el AS induce la producción de peróxido de hidrógeno $\left(\mathrm{H}_{2} \mathrm{O}_{2}\right)$ y estimula una mayor actividad de la fenilalanina amonio liasa (PAL, por sus siglas en inglés), responsable de la síntesis de compuestos fenólicos.

La relevancia en el incremento de los fenoles radica en el papel que desempeñan estos compuestos como protectores contra daños causados por plagas y enfermedades (Lattanzio et al., 2006), tales como gorgojo del maíz (Sitophilos zeamays) (García et al., 2003) y Fusarium spp. (Bakan et al., 2003). Al respecto García et al. (2003) reportaron que las bases bioquímicas de estos mecanismos de resistencia se deben en parte a los niveles elevados de compuestos fenólicos, que se encuentran enlazados con los carbohidratos de la pared celular, además de limitar la entrada de insectos y de disminuir la disponibilidad de nutrimentos; por otro lado, también se ha documentado que la acumulación intracelular de AS conlleva al aumento de los niveles de especies reactivas de oxígeno (ERO) 


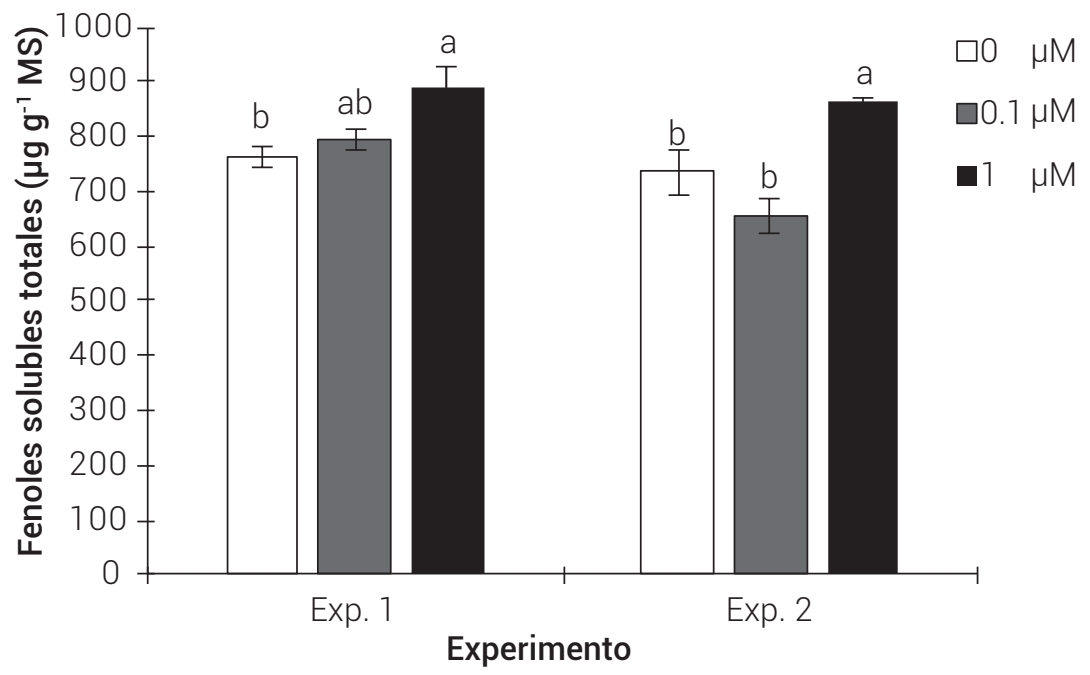

Figura 1. Concentración de fenoles totales en el grano de maíz proveniente de plantas de maíz asperjadas con dos concentraciones de ácido salicílico en fase de plántula. Cada punto es la media de cuatro repeticiones \pm error estándar. Barras con letras iguales no son estadísticamente diferentes (Tukey, 0.05).

y expresión de genes relacionados con la patogenicidad (Díaz-Puentes, 2012).

Los resultados obtenidos en esta gramínea de importancia alimenticia y económica, permiten confirmar la participación del AS en la inducción de síntesis de fenoles en las plantas (Dong et al., 2010), a la vez que reafirman su colaboración en la producción de metabolitos secundarios tal como se ha reportado en Alternanthera tenella (RodriguesBrandão et al., 2014), Saccharum officinarum y Calendula officinalis L. (Pacheco et al., 2013); además, dan la pauta para proponer su uso como herramienta en la inducción de tolerancia a plagas (García et al., 2003) y enfermedades del grano en maíz (Bakan et al., 2003), y para incrementar el poder antioxidante de este cereal, que se traduce en beneficios potenciales en la salud humana (Adom y Liu, 2002; Liu, 2007).

La aplicación foliar de ácido salicílico en concentración de $1 \mu \mathrm{M}$, en fase de plántula en maíz, incrementa la concentración de fenoles solubles totales en el grano. Aunque se conocen algunos de los compuestos fenólicos presentes en las estructuras del grano de maíz, así como su distribución y proporción de manera natural, no ha sido elucidado si el AS puede incidir en este orden o si existe preferencia en incrementar algún fenol en particular, y puesto que cada compuesto le confiere a las estructuras características muy propias para desempeñar funciones específicas (Cabrera-Soto et al., 2009; García et al., 2003), se sugiere realizar estudios adicionales sobre estos parámetros.

\section{BIBLIOGRAFÍA}

Adom K. K. and R. H. Liu (2002) Antioxidant activity of grains. Journal of Agricultural and Food Chemistry 50:6182-6187.

Al-oubaidi H. K. M. and A. S. M. Ameen (2014) Increasing secondary metabolites of Calendula officinalis L. using salicylic acid in vitro. World Journal of Pharmacy and Pharmaceutical Sciences 13:1146-1155

An C. and Z. Mou (2011) Salicylic acid and its function in plant immunity. Journal of Integrative Plant Biology 53:412-428.

Awate P. D. and D. K. Gaikwad (2014) Influence of growth regulators on secondary metabolites of medicinally important oil yielding plant Simarouba glauca DC. under water stress conditions. Journal of Stress Physiology \& Biochemistry 10:222-229.

Bakan B., A. C. Bily, D. Melcion, B. Cahagnier, C. Regnault-Roger, B. J. R. Philogène and D. Richard-Molard (2003) Possible role of plant phenolics in the production of trichothecenes by Fusarium graminearum strains on different fractions of maize kernels. Journal of Agricultural and Food Chemistry 51:2826-2831.

Cabrera-Soto M. L., Y. Salinas-Moreno, G. A. Velázquez-Cardelas y E. Espinosa T. (2009) Contenido de fenoles solubles e insolubles en las estructuras del grano de maíz y su relación con propiedades físicas. Agrociencia 43:827-839.

Díaz-Puentes L. N. (2012) Resistencia sistémica adquirida mediada por el ácido salicílico. Biotecnología en el Sector Agropecuario y Agroindustrial 10:257-267.

Dong J., G. Wan and Z. Liang (2010) Accumulation of salicylic acid-induced phenolic compounds and raised activities of secondary metabolic and antioxidative enzymes in Salvia miltiorrhiza cell culture. Journal of Biotechnology 148:99-104.

Farah A. and C. M. Donangelo (2006) Phenolic compounds in coffee. Brazilian Journal of Plant Physiology 18:23-36.

García L. S., A. J. Burt, J. A. Serratos, D. M. Díaz P., J. T. Arnason y D. J. Bergvinson (2003) Defensas naturales en el grano de maíz al ataque de Sitophilus zeamais (Motsch, Coleoptera: Curculionidae): mecanismos y bases de la resistencia. Revista de Educación Bioquímica 22:138-145.

Gutiérrez-Coronado M. A., C. Trejo-López and A. Larqué-Saavedra (1998) Effects of salicylic acid on the growth of roots and shoots in soybean. Plant Physiology and Biochemistry 36:563-565.

Hao W., H. Guo, J. Zhang, G. Hu, Y. Yao and J. Dong (2014) Hydrogen peroxide is involved in salicylic acid-elicited rosmarinic acid production in Salvia miltiorrhiza cell cultures. The Scientific World Journal. http://dx.doi.org/10.1155/2014/843764 
Hayat Q., S. Hayat, M. Irfan and A. Ahmad (2010) Effect of exogenous salicylic acid under changing environment: a review. Environmental and Experimental Botany 68:14-25.

Khan M. I. R., M. Fatma, T. S. Per, N. A. Anjum and N. A. Khan (2015) Salicylic acid-induced abiotic stress tolerance and underlying mechanisms in plants. Frontiers in Plant Science 6:462, doi: 10.3389/ fpls.2015.00462.

Lattanzio V., V. M. T. Lattanzio and A. Cardinali (2006) Role of phenolics in the resistance mechanisms of plants against fungal pathogens and insects. In: Phytochemistry: Advances in Research. F. Imperato (ed.). Research Signpost. Trivandrum. Kerala, India. pp:23-67.

Liu R. H. (2007) Whole grain phytochemicals and health. Journal of Cereal Science 46:207-219.

Makkar H. P. S. (2003) Quantification of Tannins in Tree and Shrub Foliage. A Laboratory Manual. Kluwer Academic Publishers. Dordrecht, The Netherlands. $102 \mathrm{p}$

Martín-Mex R., A. Nexticapan-Garcez and A. Larqué-Saavedra (2013) Potential benefits of salicylic acid in food production. In: Salicylic Acid. Plant Growth and Development. S. Hayat, A. Ahmad and M. N. Alyemeni (eds.). Springer. Dortdrecht, The Netherlands. pp: 299-313.

Pacheco A. C., C. da Silva C., E. S. da Silva F. and C. Cabral A. (2013) Salicylic acid-induced changes to growth, flowering and flavonoids production in marigold plants. Journal of Medicinal Plant Research 7:3158-3163.
Quiñones M., M. Miguel y A. Aleixandre (2012) Los polifenoles, compuestos de origen natural con efectos saludables sobre el sistema cardiovascular. Nutrición Hospitalaria 27:76-89.

Robbins R. J. (2003) Phenolic acids in foods: an overview of analytical methodology. Journal of Agricultural and Food Chemistry 51:2866-2887

Rodrigues-Brandão I., A. Moraes K., A. Millech E., M. Conceição L., L. do Amarante, J. A. Peters and E. J. Bolacel B. (2014) Salicylic acid on antioxidant activity and betacyan in production from leaves of Alternanthera tenella. Ciência Rural 44:1893-1898.

Salinas M. Y., C. García S., B. Coutiño E. y V. A. Vidal M. (2013) Variabilidad en contenido y tipos de antocianinas en granos de color azul/ morado de poblaciones mexicanas de maíz. Revista Fitotecnia Mexicana 36(Supl 3-A):285-294.

Sarrou E., P. Chatzopoulou, K. Dimassi-Theriou, I. Therios and A. Koularmani (2015) Effect of melatonin, salicylic acid and gibberellic acid on leaf essential oil and other secondary metabolites of bitter orange young seedlings. Journal of Essential Oil Research 27:487-496.

Tucuch H. C. J., G. Alcántar G. y A. Larqué S. (2015) Efecto del ácido salicílico en el crecimiento de la raíz y biomasa total de plántulas de trigo. Terra Latinoamericana 33:63-68.

Tucuch-Haas C. J., G. Alcántar-González, V. H. Volke-Haller, Y. Salinas-Moreno, L. I. Trejo-Téllez y A. Larqué-Saavedra (2016) Efecto del ácido salicílico sobre el crecimiento de raíz de plántulas de maíz. Revista Mexicana de Ciencias Agrícolas 7:709-716. 